\title{
Berberine Improves Insulin Sensitivity by Inhibiting Fat Store and Adjusting Adipokines Profile in Human Preadipocytes and Metabolic Syndrome Patients
}

\author{
Jing Yang, ${ }^{1}$ Jinhua Yin, ${ }^{1,2}$ Hongfei Gao, ${ }^{1}$ Linxin Xu, ${ }^{1,2}$ Yan Wang, ${ }^{1} \mathrm{Lu} \mathrm{Xu,}{ }^{2}$ and Ming $\mathrm{Li}^{2}$ \\ ${ }^{1}$ First Affiliated Hospital, Shanxi University of Medical, Taiyuan 030001, China \\ ${ }^{2}$ Endocrine Key Laboratory of the Ministry of Health, Department of Endocrinology, Peking Union Medical College Hospital, \\ Chinese Academy of Medical Sciences, 1 Shuaifuyuan, Wangfujing, Beijing 100730, China
}

Correspondence should be addressed to Ming Li, lmpeking@yahoo.com.cn

Received 9 October 2011; Revised 29 December 2011; Accepted 29 December 2011

Academic Editor: Hao Xu

Copyright (C) 2012 Jing Yang et al. This is an open access article distributed under the Creative Commons Attribution License, which permits unrestricted use, distribution, and reproduction in any medium, provided the original work is properly cited.

\begin{abstract}
Berberine is known to inhibit the differentiation of 3T3-L1 cells in vitro, improve glycemic control, and attenuate dyslipidemia in clinical study. The aim of this study was to investigate the effects of berberine on preadipocytes isolated from human omental fat and in metabolic syndrome patients treated with berberine for 3 months. We have shown that treatment with $10 \mu \mathrm{M}$ berberine resulted in a major inhibition of human preadipocyte differentiation and leptin and adiponectin secretion accompanied by downregulation of PPAR $\gamma 2, \mathrm{C} / \mathrm{EBP} \alpha$, adiponectin, and leptin mRNA expression. After 3 months of treatment, metabolic syndrome patients showed decrease in their BMI $\left(31.5 \pm 3.6\right.$ versus $\left.27.4 \pm 2.4 \mathrm{~kg} / \mathrm{m}^{2}\right)$ and leptin levels $(8.01$ versus $5.12 \mu \mathrm{g} / \mathrm{L})$, as well as leptin/adiponectin ratio and HOMA-IR. These results suggest that berberine improves insulin sensitivity by inhibiting fat store and adjusting adipokine profile in human preadipocytes and metabolic syndrome patients.
\end{abstract}

\section{Introduction}

The metabolic syndrome is a cluster of multiple metabolic diseases based on obesity and insulin resistance. Obesity leads to insulin resistance and a proatherogenic state. Therefore, the role of obesity, especially visceral (or central or abdominal) obesity, is believed to be the main physiological force resulting in disorders of glucose and lipid metabolism in metabolic syndrome [1]. However, because of the effects of insulin in fat cell differentiation and metabolism of glucose and lipids, patients who are treated by insulin, sulphonylureas, and thiazolidinediones may suffer from varying degrees of weight gain. Effects of metformin on body weight may be based on calorie intake reduction rather than energy consumption increased. The statins for regulating lipid metabolism are generally expensive and some of them have liver toxic side effects. Therefore, the search for a cost/effective drug that can not only lower blood glucose and lipids but also reduce weight for metabolic syndrome treatment has a significant importance.
Berberine is an isoquinoline derivative alkaloid isolated from many kinds of medicinal herbs, such as Hydrastis canadensis (goldenseal), Cortex Phellodendri (Huangbai), and Rhizoma Coptidis (Huanglian). It is safe and cheap and has been extensively used as an antibacterial drug [2]. Berberine has been proven to have many other pharmacological effects including antimicrobial [3], antitumor [4], anti-inflammation [5], blood glucose lowering [6], and even inhibiting chronic cocaine-induced sensitization [7]. In one recent single-blind clinical observation, the study showed that diet supplementation of some natural substances including berberine was beneficial for correcting lipid metabolism disorders and reducing cardiovascular risk factors [8]. However, the body weight reduction effect is poorly characterized in clinical study.

Pharmacokinetics of berberine indicates that adipose tissue is its main target [9]. Adipose tissue is a huge energy reserve organ. The excessive proliferation and differentiation of fat cells can lead to excessive fat accumulation in adipose tissue, resulting in obesity [10]. At the same time, fat cells can 
secrete a variety of hormones, named adipokines, through endocrine, paracrine, and autocrine mechanisms that affect energy metabolism of the body [11]. It is assumed that unfavorable changes in the secretion of adipokines, considered as an early symptom of impaired adipose tissue function, are the potential link between obesity and insulin resistance, influencing the development of metabolic syndrome [12]. Leptin and adiponectin are the key biomarkers of adipose tissue. Hyperleptinemia and hypoadiponectinemia are common in obesity. They reflect increased adiposity and may contribute to hypertension, dyslipidemia, impaired glucose metabolism, and proatherogenic state in obesity and metabolic syndrome $[13,14]$. Many studies have been published on the mechanism of berberine's effect on adipose tissue. Zhou et al. found that berberine significantly inhibited differentiation of mouse 3T3-L1 preadipocytes into fat cells [15]. In addition, it has also been shown to reduce leptin and resist secretion [16] and increase the mRNA expression of adiponectin [17]. Members in our research team, Zhang et al. also found berberine-moderated glucose and lipid metabolism through a multipathway mechanism that includes AMP-activated protein kinase- (AMPK-) p38 MAPK-GLUT4, JNK pathway, and PPAR $\alpha$ pathway in KKAy mice [18]. These results showed that berberine may have excellent potential as an agent to prevent metabolic syndrome. However, these studies were performed in rodent models or murine cell lines. The effects of berberine on human adipose tissue are rarely reported. Due to lack of well-established human adipocyte model, human primarily cultured preadipocytes have been particularly useful for verifying the results obtained from the preadipocyte cell lines. Thus, in this paper, we presented evidence obtained from human primarily cultured omental preadipocytes as well as from metabolic syndrome patients and demonstrated that berberine improves insulin sensitivity by inhibiting fat store and adjusting the profile of adipokines.

\section{Materials and Methods}

2.1. Materials. Berberine used in vitro study was purchased from Sigma Aldrich Co, St. Luis, MO, USA. Oral medication berberine used in vivo study has Chinese Drug Approval Number: H.M.L.N., H11022584.

2.2. Adipose Biopsies. Omental adipose tissue biopsies were obtained from nine patients ( 3 females, 6 males, age range $22 \sim 47$ years) who underwent elective inguinal hernia repair surgery. None of these patients suffered from endocrine malignant or chronic inflammatory diseases or severe systemic illnesses or any recent weight change. None were taking medications known to affect adipose tissue mass or metabolism. The study was approved by the local ethical committee. All patients gave their informed consent. On the day of surgery, all patients fasted for at least $6 \mathrm{~h}$ preoperatively, and all underwent general anesthesia. Adipose tissue specimens from the omental adipose tissue regions were obtained within 30-45 min after the onset of surgery. In general, 10-15 $\mathrm{g}$ of adipose tissue was obtained and transported to the laboratory in normal saline (transport time with $10 \mathrm{~min}$ ). Specimens from three patients were used for cell proliferation experiments and six for cell differentiation studies.

2.3. Cell Culture. The isolation and culture of preadipocytes was performed according to the method described elsewhere by ourselves [19]. Briefly, Adipose tissue was cut into $1 \mathrm{~mm} \times$ $1 \mathrm{~mm}$ pieces with ophthalmic scissors. Collagenase digestion was performed at $37^{\circ} \mathrm{C}$ on a shaking platform $(200 \mathrm{rpm})$ for 1 to 3 hours. Next, digest was transferred to filter by $74 \mu \mathrm{m}$ sieve size filter. This procedure was repeated until the complete digestion was filtered. The cell suspension was centrifuged at $480 \mathrm{~g}$ for $5 \mathrm{~min}$, and the preadipocyte fraction was resuspended in growth medium (PromoCell, Germany). Then cells were counted and cultured in different mediums at $37^{\circ} \mathrm{C}$ in a humidified $5 \% \mathrm{CO}_{2}$ atmosphere.

2.4. Analysis of Cell Proliferation. $4 \times 10^{3}$ preadipocytes/well were inoculated into 96 -well plates and cultured in growth medium supplemented with varying concentrations of berberine $(0 \mu \mathrm{M}, 0.1 \mu \mathrm{M}, 1 \mu \mathrm{M}$, and $10 \mu \mathrm{M})$. Proliferation was determined by MTT assay after 1,2, and 3 days of culture. Briefly, after culture medium was removed, MTT $(0.5 \mathrm{mg} / \mathrm{mL}, 50 \mu \mathrm{L} /$ well $)$ was added into the plates and incubated at $37^{\circ} \mathrm{C}$ for $4 \mathrm{~h}$, followed by the addition of DMSO $(150 \mu \mathrm{L} /$ well $)$, and incubated at $37^{\circ} \mathrm{C}$ for 1 hour. The proliferation values were obtained from the optical density (OD) measured at $570 \mathrm{~nm}$ with $650 \mathrm{~nm}$ as background. Data are presented as percentage of the untreated controls $(0 \mu \mathrm{M}$ berberine) at each time point.

2.5. Analysis of Cell Differentiation. $5 \times 10^{4}$ cells/well cells were inoculated in 24-well plates. After 48 hours, cells were induced to differentiate in differentiation medium (PromoCell, Germany) containing varying concentrations of berberine $(0 \mu \mathrm{M}, 0.1 \mu \mathrm{M}, 1 \mu \mathrm{M}$, and $10 \mu \mathrm{M})$. After 16 days, the degree of differentiation was determined by Oil-RedO staining performed as previously reported [20]. In brief, medium was removed and cells were washed with PBS twice, fixed with $3.7 \%$ formalin at room temperature for $30 \mathrm{~min}$, added 60\% 2-propanol and incubated for $5 \mathrm{~min}$, then moved out 2-propanol and stained cells with Oil-Red-O solution (Sigma, USA) at room temperature for $10 \mathrm{~min}$. Images were obtained using an Olympus IX70 inverted phase-contrast microscopy (Olympus, Japan). After staining, the cells were washed twice with 70\% ethanol and dissolved in 2-propanol containing 4\% Nonidet-P40. OD values were measured at an absorbance of $490 \mathrm{~nm}$ using a standard microtiter reader (Bio-Rad, Canada).

2.6. RT-PCR Analysis. $5 \times 10^{5}$ cells/well cells were seeded in 6 -well plates. 8 days after differentiation, $10 \mu \mathrm{M}$ berberine was added in differentiation medium. Cells were harvested for 24 hours afterwards and mRNAs were extracted with Trizol reagent (Invitrogen, USA). RNA recovery and quality were checked by measuring the $260 / 280 \mathrm{~nm}$ optical density ratio and by electrophoresis on $1.5 \%$ agarose gel. $1 \mu \mathrm{g}$ of total RNA from each sample was used for reverse transcription reaction using the TaqMan reverse transcription 
reagents (Applied Biosystems, USA). The expression levels of peroxisome proliferator-activated receptor $\gamma 2$ (PPAR $\gamma 2)$, CCAAT enhancer-binding protein $\alpha(\mathrm{C} / \mathrm{EBP} \alpha)$, lipoprotein lipase, leptin, and adiponectin were measured using the following oligonucleotides (Shanghai Biotechnology Engineering Service Co. Ltd., China): 5'-GTG/GGG/CGC/CCC/AGG/CAC/CA-3' and $3^{\prime}$-CTT/TAG/CAC/GCA/CTG/T$\mathrm{AA} / \mathrm{TTC} / \mathrm{CTT} / \mathrm{C}-5^{\prime}$ primers for $\beta$-actin; $5^{\prime}$-ACC/CTG/TGC/GGA/TTC/TTG/TGG/CTC/TGT- $3^{\prime}$ and $3^{\prime}$-CGA/AGT/CCG/ATG/AGG/TGT/CTC-5' primers for leptin; $5^{\prime}$ CTG/GGA/GCT/GTT/CTA/CTG/C-3' and 3'-AGT/CAC/CCT/AAC/CTC/GT-5' primers for adiponectin; 5'-GCG/ATT/CCT/TCA/CTG/ATA/CAC-3' and 3'-CGG/ACG/TAG/AGG/TGG/AAT/AAT-5' primers for PPAR $\gamma 2 ; 5^{\prime}$-GCA/AGG/CCA/AGA/AGT/CGG/TGG/AC-3' and $3^{\prime}$-GAG/GAA/CCA/GTT/CCG/GTA/CCC/GT- $5^{\prime}$ primers for C/EBP $\alpha$; $5^{\prime}$-ACA/CAG/CTG/AGG/ACA/CTT/GC-3' and $3^{\prime}$-GAG/TCC/TCG/TAA/TGG/GTC/AC-5' primers for lipoprotein lipase. The basic reaction conditions are as follows: DNA denaturation at $94^{\circ} \mathrm{C}$ for $5 \mathrm{~min}$; PCR amplification: $94^{\circ} \mathrm{C}$ denaturation for $50 \mathrm{sec}$, specific annealing temperature for $50 \mathrm{sec}, 72^{\circ} \mathrm{C}$ extension for $1 \mathrm{~min}$, and final extension also at $72^{\circ} \mathrm{C}$ for $8 \mathrm{~min}$. To ensure that amplification of these products was within the exponential range, different numbers of PCR cycles (25-40 cycles) were run. PCR products were sent to Shanghai Biotechnology Engineering Service Co. Ltd. for sequence verification. PCR products were analyzed on a $2 \%$ agarose gel, and semiquantitative analysis was performed (quantification with Bio-1D software, France).

2.7. Effects of Berberine on Secreted Proteins in the Human Preadipocyte Differentiation Process. $10^{5}$ cells/well cells were inoculated in 12-well plates. Cells were induced with differentiation medium. Beginning on the third day, supernatants were collected every 2 days and the final collections were done after 21 days of differentiation. Leptin and adiponectin proteins were measured using commercial ELISA kits (Quantikine, R\&D Systems, Germany). The intra- and interassay CVs for leptin were $<3.3 \%$ and $<5.4 \%$, respectively; the intra- and interassay CVs for adiponectin were $<5.0 \%$ and $<7.9 \%$, respectively.

2.8. Clinical Intervention Study. 41 patients (age ranged 32 68 years) with newly diagnosed metabolic syndrome enrolled in this study. 3 of them were initiative to withdraw from the study on medication 1, 3, and 6 days, and 1 person lost contact. In the end, 37 people (17 males/20 females) finished the clinical trials. Metabolic syndrome was defined according to Chinese Diabetes Society definition set in 2004 [21]. The study had the approval of the local ethical committee, and informed consent was obtained from all patients. Patients were treated with berberine $0.3 \mathrm{~g}$ three times a day for 12 weeks, and the following indicators before and after treatment were measured: height, weight, waist circumference, fasting plasma glucose, fasting insulin, hemoglobin A1C (HbAlc), triglyceride, cholesterol, LDL cholesterol, high-density lipoprotein, adiponectin, and leptin. The following were calculated: BMI, Leptin/Adiponectin ratio, and homeostasis model of assessment insulin resistance index $[$ HOMA-IR $=$ fasting insulin $(\mathrm{mIU} / \mathrm{L}) \times$ fasting glucose $(\mathrm{mmol} / \mathrm{L}) / 22.5]$.

2.9. Statistical Analysis. Descriptive statistics and analysis were performed in SPSS 13.0 for Windows (SPSS Inc. Chicago, IL). $t$-tests of two independent samples were done to determine the mean comparison in cell study (test of homogeneity of variance, such as $P<0.10$, line $t$-test), and $t$-test of paired measurement data was done in clinical study before and after medication. Data of normal distribution were expressed as means \pm the standard deviation. Data of nonnormal distribution were expressed as median $(\mathrm{M})$ and quartile (Q1/4). The $\alpha$ level was set at 0.05 .

\section{Results}

3.1. Omental Preadipocytes and Induced Mature Adipocytes. Human omental preadipocytes were isolated and primarily cultured in growth medium. After 3-4 days, these cells began to show the typical long spindle shape (Figure 1(a)) and started to proliferate. Preadipocytes were induced to differentiate, and morphological changes can be observed after 15 days. When preadipocytes gradually mature, their cytoplasm was filled with lipid droplets, and small lipid droplets were integrated into big lipid droplets (Figure 1(b)). Fat droplets in adipocytes can be observed in the cytoplasm by Oil-Red-O staining (Figure 1(c)).

3.2. Effect of Berberine on Human Preadipocyte Proliferation. Primary human omental preadipocytes were treated with different concentrations of berberine and OD values were measured at day 1 , day 2 , or day 3 . Results show that the relative $\mathrm{OD}$ values are significantly higher when berberine was added at $0.1 \mu \mathrm{M}, 1 \mu \mathrm{M}$, and $10 \mu \mathrm{M}$ concentrations compared with the control group $(P<0.05)$ (Figure 2). No significant difference was found among berberine-treated groups $(P>0.05)$.

\subsection{Effect of Berberine on Human Preadipocyte Differentia-} tion. Different concentrations of berberine were used during the cell differentiation process, and cells' morphological changes through Oil-Red-O staining were observed at day 16. Cell density was reduced judging by the stained color per increasing drug concentrations at low magnification field of vision. This suggests that berberine inhibits the process of cell differentiation and hypertrophy (Figure 3(a)). The staining intensity was measured. OD values of the berberine groups at concentrations of $1 \mu \mathrm{M}$ and $10 \mu \mathrm{M}$ were significantly lower than of the control groups, and the decreases determined were dose dependent $(P<0.05)$ (Figure 3(b)).

3.4. Effect of Berberine on PPAR 2 , Lipoprotein Lipase, $C / E B P \alpha$, Leptin, and Adiponectin mRNA Expression. Preadipocytes were induced to differentiate over 8 days, then $10 \mu \mathrm{M}$ berberine was added and cells were harvested for 24 hours. The expression levels of PPAR $\gamma 2$, lipoprotein lipase, $\mathrm{C} / \mathrm{EBP} \alpha$, leptin, and adiponectin were measured by RTPCR. For quality control, the resulting PCR products were sequenced in duplicate and showed $>85 \%$ homology with 


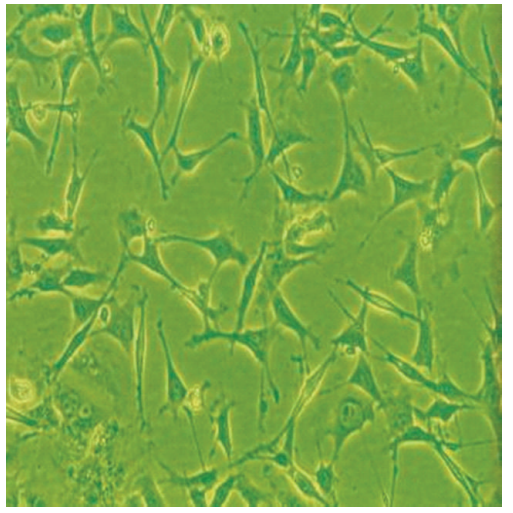

(a)

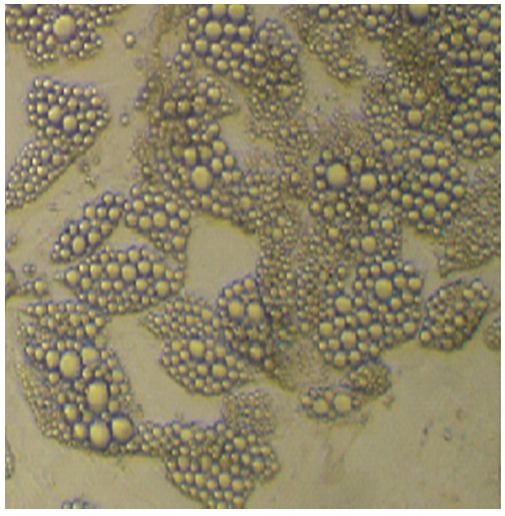

(b)

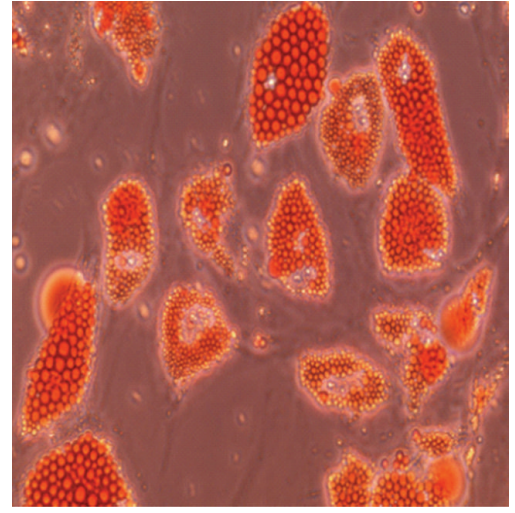

(c)

FIGURE 1: Representative phase-contrast images of human omental preadipocytes in primary culture and differentiated preadipocytes. (a) Human omental preadipocytes in primary culture, (b) mature adipocytes induced from preadipocyte differentiation, and (c) mature adipocytes stained with Oil-Red-O, $\times 200$.

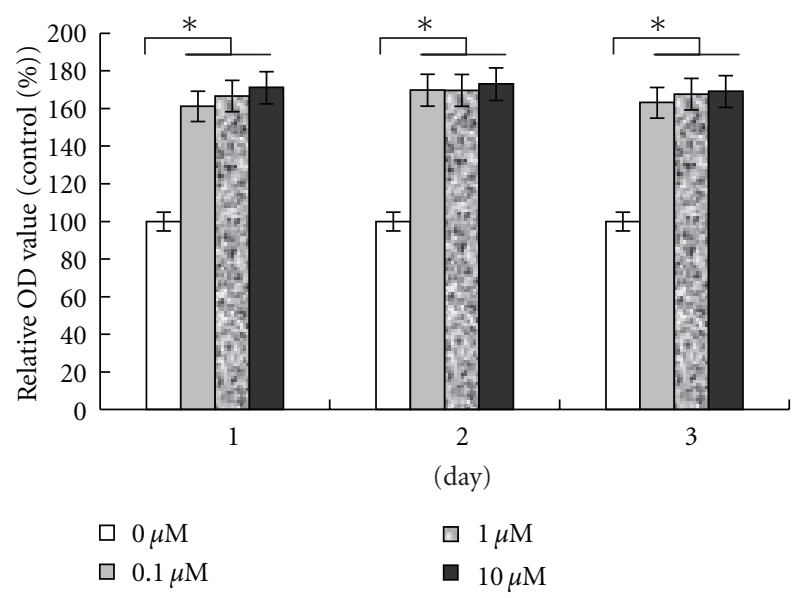

FIGURE 2: Effect of berberine on human preadipocyte proliferation. Cells were cultured in growth medium with different concentrations of berberine for 1, 2, and 3 days. At each culture time point, proliferation capacity was determined by MTT assay. Values are expressed as percentage of the untreated controls and represent the mean \pm SEM of the three separate experiments in eight replicates. ${ }^{*} P<0.05$, compared to control at each time point.

GenBank registration sequences. Comparison of the ratio of the gray degree between the specific gene band and internal reference $\beta$-actin band showed that $10 \mu \mathrm{M}$ of berberine inhibits $\operatorname{PPAR} \gamma 2$, lipoprotein lipase, $\mathrm{C} / \mathrm{EBP} \alpha$, adiponectin, and leptin mRNA expression (Figures 4(a) and 4(b)).

\subsection{Effect of Berberine on Leptin and Adiponectin Secretion} during the Process of Preadipocyte Differentiation. When preadipocytes were cultured with growth medium, a very low level of leptin protein was detected. However, the levels did not change with time. No adiponectin secretion can be detected. Once preadipocytes were cultured in differentiation medium, the secretion of leptin increased gradually with time. It increased much more rapidly after day 9 and reached the peak at day $17 \sim 19$. After that, it maintained a high level of secretion. The secretion of adiponectin was also differentiation induced, and at day 7 , low levels of secretion can be detected. This was the time when fat cells containing lipid droplets can be seen under microscope. After 15 17 days, adiponectin secretion reached its peak; however, it began to decrease significantly at day $21(P<0.05)$. For the $10 \mu \mathrm{M}$ berberine-treated differentiation medium group, the levels of leptin and adiponectin secretion were significantly reduced starting from day 9 and remained low until day 21 (from mid- to terminal stages of differentiation) (Figure 5).

3.6. Effects of Berberine on the Impact Clinical Markers. Patients with newly diagnosed metabolic syndrome were treated with berberine for 12 weeks. Their BMI, waist circumference, fasting plasma glucose, fasting insulin, HbA1c, triglyceride, total cholesterol, LDL cholesterol, leptin, the ratio of leptin and adiponectin, and HOMA-IR were measured before and after the treatment. All of those indexes showed a decreasing trend, with a significant statistical difference $(P<0.05-0.01)$. However, the levels of adiponectin did not change significantly (Table 1 ).

3.7. Safety. None of the patients experienced severe gastrointestinal adverse events when berberine was used. Incidence of gastrointestinal adverse events was mainly constipation $(n=$ 1 ; percentage, $5 \%$ ). The adverse effect disappeared when berberine dosage was decreased from $0.3 \mathrm{~g}$ three times a day to $0.2 \mathrm{~g}$ three times a day. Liver and kidney functions were monitored in this study. No significant changes of plasma ALT, $\gamma$-GT, and creatinine were observed during the 12 weeks of berberine treatment (Table 1). None of the patients were observed with hypoglycemia.

\section{Discussion}

Proliferation and differentiation are two important aspects in fat tissue development. In our experiment, we have shown that berberine has a growth-factor-like role on human preadipocytes, promoting cell proliferation. Our result is 


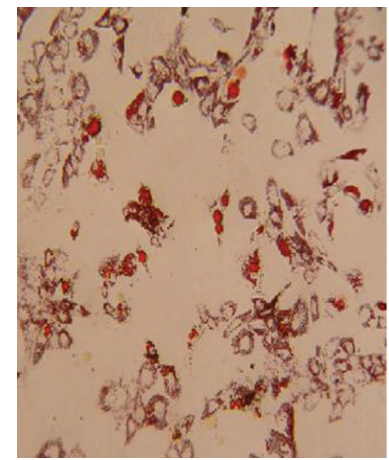

(A)

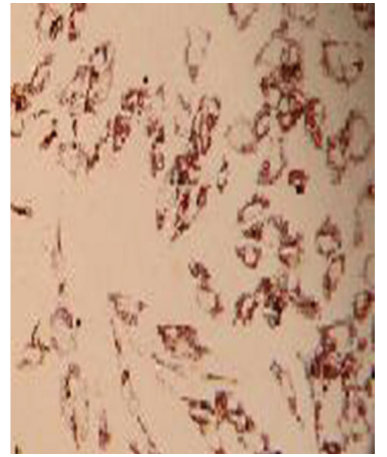

(B)

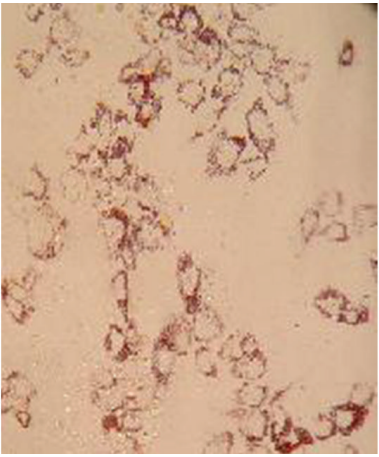

(C)

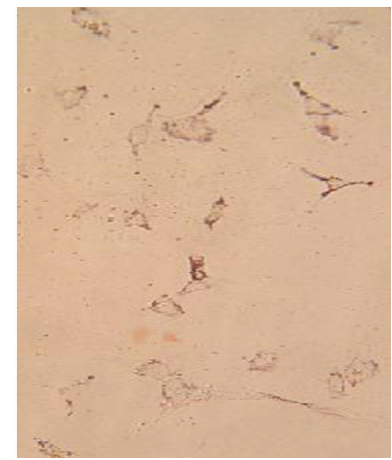

(D)

(a)

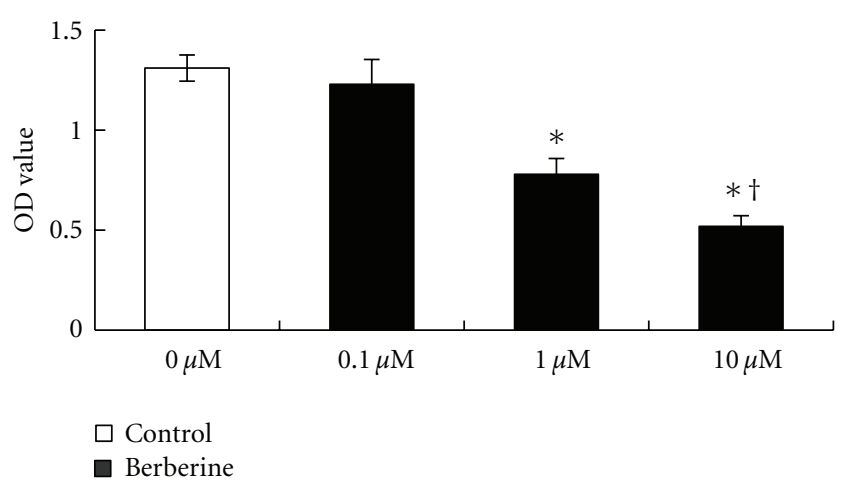

(b)

Figure 3: Effect of berberine on human preadipocyte differentiation. Cells differentiated in the absence or presence of different concentrations of berberine over 16 days. The degree of differentiation was determined by Oil-Red-O staining. (a) Photomicrographs representing cells maintained in different concentrations of berberine: (A) control, (B) $0.1 \mu \mathrm{M}$ berberine, (C) $1 \mu \mathrm{M}$ berberine, (D) $10 \mu \mathrm{M}$ berberine, $\times 100$. (b) Absorbance value representing the mean \pm SEM of the three separate experiments in six replicates. ${ }^{*} P<0.01$, comparison between berberine-treated groups and control group, ${ }^{\dagger} P<0.05$, comparison among berberine-treated groups.

consistent with previous studies showing that berberine can promote the proliferation of mouse 3T3-L1 preadipocyte [22]. Adult obesity is mainly due to the increased volume of fat cells which are abnormally hypertrophic [23]. Our in vitro experiments showed that berberine significantly inhibited the omental preadipocytes to become mature adipocytes judging by their morphology or lipid-specific OilRed-O staining. Therefore, berberine has potential clinical application in reducing visceral fat and controlling central obesity. It has been reported that fat tissue composed of a higher amount of small fat cells is more sensitive to insulin compared with fat tissue with the same lipid content composed of a small number of large fat cells, and also the former has very little inflammatory responses [24]. Our results showed that berberine can promote human fat cell proliferation and inhibit fat cell enlargement, indicating that it may be able to reduce inflammation responses, improve insulin sensitivity of visceral adipose tissue, and reduce or eliminate the visceral adipose tissue. Moreover, our in vivo study also showed that, after taking berberine for three month, patients with metabolic syndrome were found to reduce their waist circumferences and BMI to varying degrees. This positive result therefore seems in good agreement with the in vitro study.
The nuclear receptor PPAR $y$ and members of the C/EBP family take important roles in adipogenesis [25], and the major players are PPAR $\gamma 2$ [26] and $\mathrm{C} / \mathrm{EBP} \alpha$ [27]. Many studies have showed that berberine inhibited the mRNA and protein levels of adipogenesis-related transcription factors PPAR $\gamma 2$ and $\operatorname{C/EBP} \alpha[28,29]$. We studied the effect and transcriptional impact of berberine on human preadipocyte differentiation. Our result showed that the berberine can inhibit PPAR $\gamma 2$ and $\mathrm{C} / \mathrm{EBP} \alpha$ mRNA expression simultaneously during the human preadipocyte differentiation process. Recently, the transcription factors GATA binding protein 2 and 3 (GATA-2 and GATA-3) have been shown to be important gate keepers of the differentiation process [30, 31]. Studies from $\mathrm{HU}$ et al. showed that berberine increases expression of GATA-2 and GATA-3 during inhibition of adipocyte differentiation in both murine cell lines 3T3-L1 and human white preadipocytes cell line. But contradictorily, they also found that the differentiation inhibition mechanisms of berberine appeared to be independent of $\operatorname{PPAR} \gamma 2$ and $\mathrm{C} / \mathrm{EBP} \alpha$ in that human white preadipocyte cell line while being dependent on decreasing of PPAR $\gamma 2$ and $\mathrm{C} / \mathrm{EBP} \alpha$ gene expression in 3T3-L1 lines $[32,33]$. Those results also seem to contrast with our findings from human primarily cultured preadipocytes. Since there is still lack 


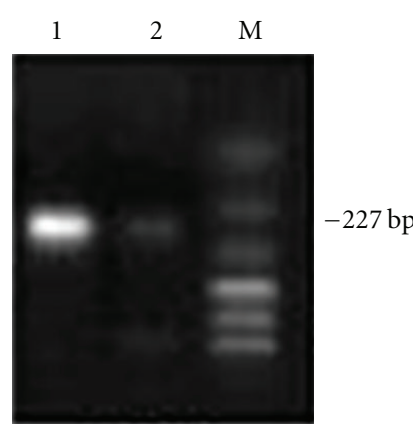

LPL

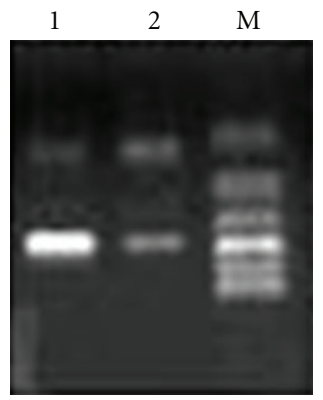

Adiponectin

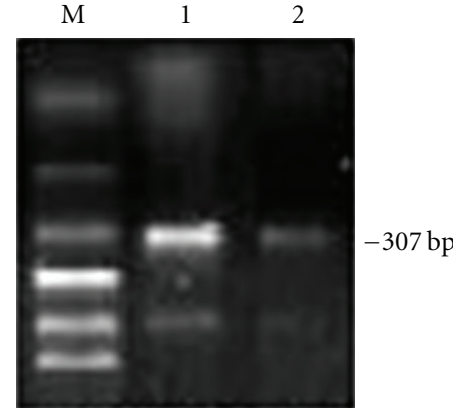

PPAR $\gamma 2$

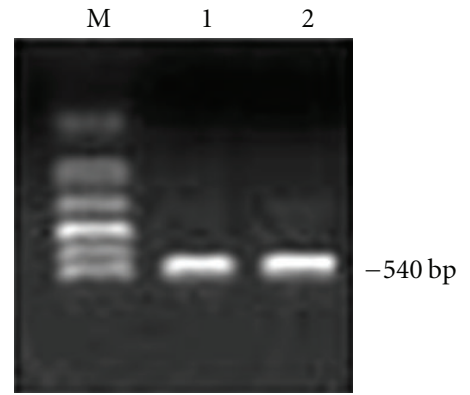

$\beta$-actin

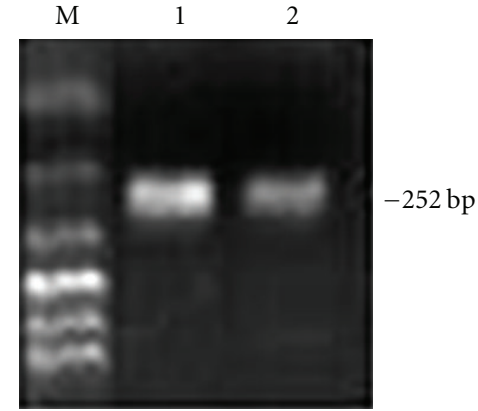

$\mathrm{C} / \mathrm{EBP} \alpha$

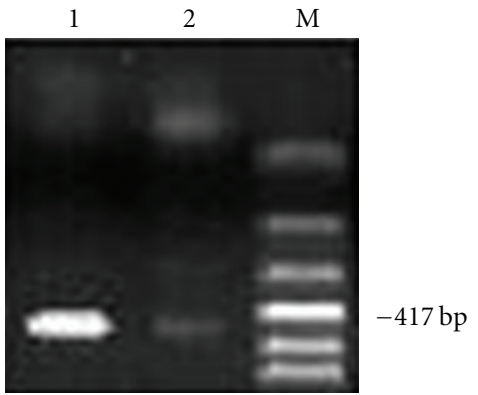

Leptin

(a)

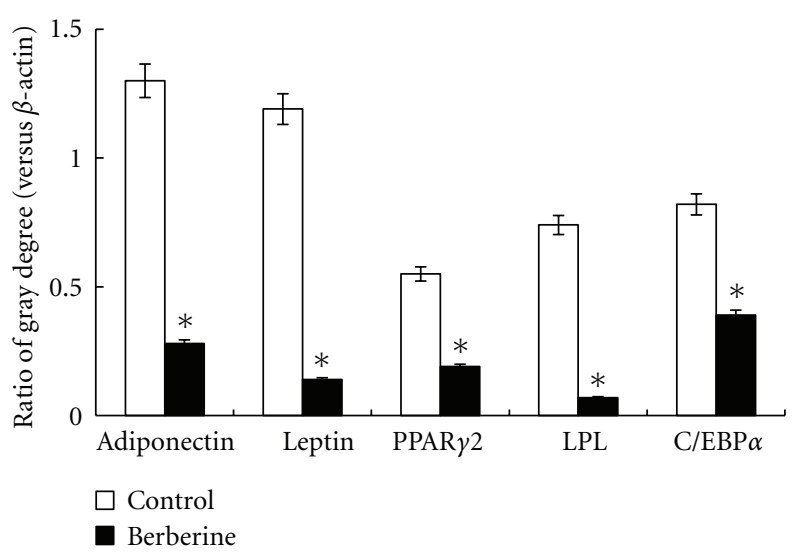

(b)

Figure 4: Effect of berberine on PPAR $\gamma 2$, lipoprotein lipase, $\mathrm{C} / \mathrm{EBP} \alpha$, leptin, and adiponectin mRNA expression in differentiated preadipocytes analyzed using RT-PCR. (a) Digital photos of PCR products in agarose gel. Lane 1: control group, lane 2: $10 \mu \mathrm{M}$ berberine, lane M: DNA markers. (b) Results are expressed as the ratio between the intensity of band corresponding to target gene versus that to $\beta$-actin, representing the mean \pm SEM of the three separate experiments in triplicate. ${ }^{*} P<0.05$, compared to control.

of well-characterized human preadipocyte cell lines, further studies in human primarily cultures are particularly needed to clarify the results from those cell lines.

Lipoprotein lipase is a kind of glycoprotein synthesized and secreted by fat cells. Current understandings of lipoprotein lipase's physiological functions are to decompose chylomicrons and triglycerides into very low-density lipoproteins and to promote the lipoprotein transfer between triglyceride phospholipids and apolipoproteins and so forth. We found that berberine reduced lipoprotein lipase mRNA expression in human fat cells which is consistent with the recent study by Choi et al. showing that berberine reduces mouse 3T3-L1 lipoprotein lipase mRNA expression [9]. As discussed by Kong et al. [34], oral administration of berberine in 32 hypercholesterolemia patients for 3 months reduced serum cholesterol by $29 \%$, triglycerides by $35 \%$, and LDL cholesterol by $25 \%$. Our clinical study of the effects of berberine on total cholesterol, triglycerides, and LDL cholesterol along with analysis of liver and kidney adverse reactions also indicates that berberine could be a cheap, efficient, and safe lipid-lowering drug in metabolic syndrome patients.

Leptin and adiponectin have been shown to play an important role in insulin resistance. During the process of 
TABLE 1: The general information and laboratory data of the new diagnosed metabolic syndrome patients at the baseline and 12 weeks after the therapy.

\begin{tabular}{|c|c|c|c|}
\hline & Before medication & After medication & $P$ value \\
\hline Participants (M/F) & $37(17 / 20)$ & $37(17 / 20)$ & \\
\hline Age (years) & $41.1 \pm 7.3$ & $41.1 \pm 7.3$ & \\
\hline Body mass index $\left(\mathrm{kg} / \mathrm{m}^{2}\right)$ & $31.5 \pm 3.6$ & $27.4 \pm 2.4$ & $<0.01$ \\
\hline Waist circumference $(\mathrm{cm})$ & $97.3 \pm 10.5$ & $92.1 \pm 9.10$ & 0.04 \\
\hline Systolic pressure (mmHg) & $146.1 \pm 14.9$ & $134.0 \pm 13.3$ & 0.32 \\
\hline Diastolic pressure (mmHg) & $95.5 \pm 8.7$ & $88.5 \pm 10.3$ & 0.24 \\
\hline Total cholesterol (mM) & $6.69 \pm 1.04$ & $5.74 \pm 0.84$ & 0.03 \\
\hline High-density lipoprotein cholesterol (mM) & $1.09 \pm 0.36$ & $0.92 \pm 0.36$ & 0.06 \\
\hline Low-density lipoprotein cholesterol (mM) & $3.68 \pm 0.85$ & $2.86 \pm 0.57$ & 0.03 \\
\hline Triglyceride (mM) & $3.03 \pm 2.05$ & $1.86 \pm 0.90$ & $<0.01$ \\
\hline Fasting plasma glucose (mM) & $7.37 \pm 0.72$ & $6.13 \pm 0.85$ & 0.03 \\
\hline HbAlc $(\%)$ & $7.10 \pm 0.64$ & $6.04 \pm 0.62$ & 0.02 \\
\hline Leptin $(\mathrm{ug} / \mathrm{L})$ & $8.01(2.04 \sim 15.17)$ & $5.12(1.88 \sim 12.89)$ & 0.04 \\
\hline Adiponectin (mg/L) & $8.42(4.75 \sim 14.81)$ & $10.02(5.13 \sim 15.59)$ & 0.14 \\
\hline Leptin/adiponectin & $0.76(0.29 \sim 2.85)$ & $0.58(0.14 \sim 1.22)$ & 0.02 \\
\hline Fasting insulin (mIU/L) & $16.90(11.6 \sim 20.1)$ & $12.50(9.7 \sim 14.8)$ & 0.04 \\
\hline HOMA-IR & $5.46(3.62 \sim 6.69)$ & $3.25(2.50 \sim 4.58)$ & 0.03 \\
\hline Glutamic-pyruvic transaminase (U/L) & $37.28 \pm 4.12$ & $39.89 \pm 7.08$ & 0.62 \\
\hline$\gamma$-Glutamyl transpeptidase (U/L) & $48.71 \pm 8.12$ & $41.79 \pm 7.11$ & 0.74 \\
\hline Creatinine $(\mathrm{mM})$ & $87.45 \pm 4.71$ & $89.11 \pm 8.07$ & 0.77 \\
\hline
\end{tabular}

$t$-test of paired measurement data comparisons between two comparing lines before and after medication. Data of nonnormal distribution were described using the median $(\mathrm{M})$ and quartile $(\mathrm{Q} 1 \sim \mathrm{Q} 4)$.

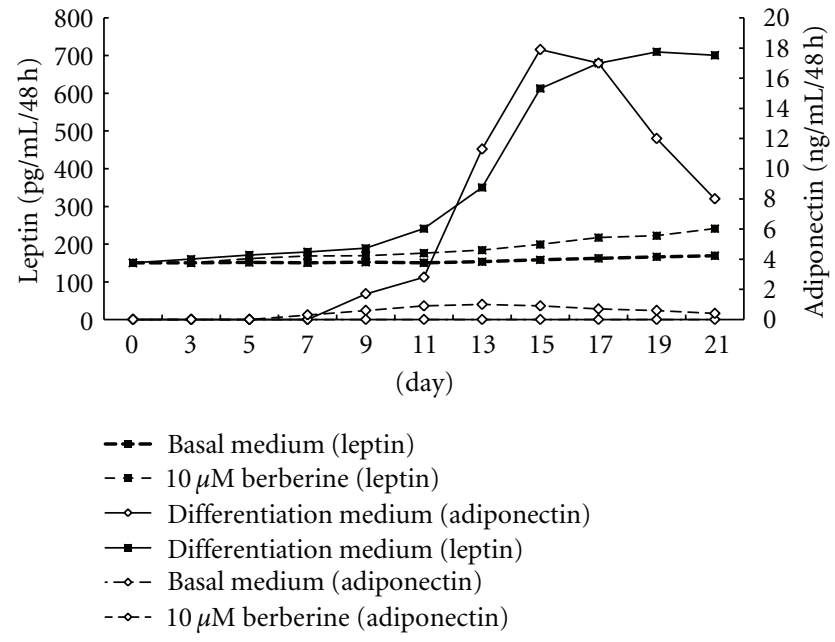

FIGURE 5: Effect of berberine on leptin and adiponectin secretion during the process of preadipocyte differentiation. Results represent the mean values of the three separate experiments in triplicate.

preadipocytes differentiation, secretion of leptin and adiponectin showed different kinetics: in the first phase low levels of leptin secretion can be detected in fat cells, but no adiponectin secretion can be detected. The amount of leptin secretion continued to increase in a synchronized fashion with the differentiation process, while the secretion of adiponectin was only observed when preadipocytes gradually mature and their cytoplasm began to be filled with lipid droplets. This result suggests that only mature fat cells can secrete adiponectin which can be used as a specific marker to determine the maturity of fat cells. In the midstages of differentiation, secretion of leptin and adiponectin increased with an increased number of fat cells; however, the rate of increase in adiponectin was more obvious. In the late stages of differentiation (at day 17-21), a morphological change of the cells showed that the majority of cells differentiated into fat cells with their cytoplasm filled with large lipid droplets. Oil-Red-O staining showed that lipid content in the cytoplasm remained at a steady high level. At this point, leptin secretion remained at high levels, while adiponectin secretion was seen to show a clearly downward trend. This difference suggests that fat cells in different fat-storing states secrete leptin and adiponectin differently, which may also reflect on their functional differences. The differentiation stages of fat cells from clinically obese patients may be different from normal people. In obese patients, the fat cells may show a high-leptin and low-adiponectin secretion pattern. In our differentiation experiment, berberine not only inhibited the differentiation and maturation of preadipocytes but also suppressed leptin and adiponectin secretion. This suggests to us that, in the visceral adipose tissue accumulation process, berberine has a role in endocrine function regulation and can promote the reversion of the initial process of fat storing. Our clinical observation showed that in patients with newly diagnosed metabolic syndrome the level of leptin dropped 
significantly with berberine treatment after 3 months. This is different from results shown in rat studies and may reflect species differences. This may suggest that berberine has the potential for anticentral obesity and regulating obesityrelated endocrine dysfunctions, thus achieving a balance between fat cell factors. Studies have reported that the ratio of leptin and adiponectin reflects the body's insulin resistance [35]. We have confirmed that berberine reduces HOMAIR and the ratio of leptin and adiponectin. As it inhibits PPAR $\gamma 2$ mRNA expression and has more effects on weight loss and reducing leptin levels, berberine regulates insulin sensitivity with a mechanism different from the insulin sensitizer, thiazolidinediones. Thus, berberine provides an additional way for clinical treatment of metabolic syndrome and obesity-related diseases. However, our experiment is only a preliminary study on the mechanisms of effects of berberine on serum adipokines. A large-scale clinical study is ideally required to include different population groups and more experiments concerning the mechanism details.

In conclusion, in order to explore the mechanism of berberine's role in improving insulin sensitivity, we used human adipose tissue as material, focusing on the proliferation, differentiation, and adipokine secretion of human preadipocytes. We tried to find clues from the in vitro experiments and then verified them in clinical trial. Our clinical study has some limitations relative to the randomized, placebo-controlled clinical design. However, our result is in agreement with the findings from previous large sample, well-designed clinical studies $[23,34]$, indicating that berberine improves glucose and lipid metabolism disorders. More particularly, we find that berberine can improve insulin sensitivity by adjusting adipokine secretion both in primarily cultured preadipocytes as well as in metabolic syndrome patients, and this was not well characterized in previous human studies.

\section{Conflict of Interests}

The authors declare that there is no conflict of interests.

\section{Acknowledgments}

The authors thank all the participants. Grants from Key Laboratory of Endocrinology Ministry of Health and Peking Union Medical College Hospital, New Star Project of Beijing Science and Technology (2004A027), and National Natural Science Foundation of China (30872165) are Acknowledged.

\section{References}

[1] R. H. Eckel, S. M. Grundy, and P. Z. Zimmet, "The metabolic syndrome," The Lancet, vol. 365, no. 9468, pp. 1415-1428, 2005.

[2] K. C. Huang and W. M. Williams, "Antibacterial, antiviral, and antifungal herbs," Pharmacology of Chinese Herbs, vol. 8, no. 6, pp. 381-383, 1999 (Chinese).

[3] A. H. Amin, T. V. Subbaiah, and K. M. Abbasi, "Berberine sulfate: antimicrobial activity, bioassay, and mode of action," Canadian Journal of Microbiology, vol. 15, no. 9, pp. 10671076, 1969.
[4] K. V. Anis, N. V. Rajeshkumar, and R. Kuttan, "Inhibition of chemical carcinogenesis by berberine in rats and mice," Journal of Pharmacy and Pharmacology, vol. 53, no. 5, pp. 763768, 2001.

[5] C. L. Kuo, C. W. Chi, and T. Y. Liu, "The anti-inflammatory potential of berberine in vitro and in vivo," Cancer Letters, vol. 203, no. 2, pp. 127-137, 2004.

[6] H. Zhang, J. Wei, R. Xue et al., "Berberine lowers blood glucose in type 2 diabetes mellitus patients through increasing insulin receptor expression," Metabolism, vol. 59, no. 2, pp. 285-292, 2010.

[7] B. Lee, C. H. Yang, D. H. Hahm et al., "Inhibitory effects of Coptidis rhizoma and berberine on cocaine-induced sensitization," Evidence-Based Complementary and Alternative Medicine, vol. 6, no. 1, pp. 85-90, 2009.

[8] A. F. G. Cicero, L. C. Rovati, and I. Setnikar, "Eulipidemic effects of berberine administered alone or in combination with other natural cholesterol-lowering agents: a single-blind clinical investigation," Arzneimittel-Forschung, vol. 57, no. 1, pp. 26-30, 2007.

[9] B. H. Choi, I. S. Ahn, Y. H. Kim et al., "Berberine reduces the expression of adipogenic enzymes and inflammatory molecules of 3T3-L1 adipocyte," Experimental and Molecular Medicine, vol. 38, no. 6, pp. 599-605, 2006.

[10] D. A. K. Roncari, "Abnormalities of adipose cells in massive obesity," International Journal of Obesity, vol. 14, supplement 3, pp. 187-192, 1990.

[11] E. E. Kershaw and J. S. Flier, "Adipose tissue as an endocrine organ," Journal of Clinical Endocrinology and Metabolism, vol. 89, no. 6, pp. 2548-2556, 2004.

[12] S. A. Ritchie and J. M. C. Connell, "The link between abdominal obesity, metabolic syndrome and cardiovascular disease," Nutrition, Metabolism \& Cardiovascular Diseases, vol. 17, no. 4, pp. 319-326, 2007.

[13] S. Younus and G. Rodgers, "Biomarkers associated with cardiometabolic risk in obesity," The American Heart Hospital Journal, vol. 9, no. 1, pp. E28-E32, 2011.

[14] M. E. Trujillo and P. E. Scherer, "Adiponectin-journey from an adipocyte secretory protein to biomarker of the metabolic syndrome," Journal of Internal Medicine, vol. 257, no. 2, pp. 167-175, 2005.

[15] L. B. Zhou, M. D. Chen, X. Wang et al., "Effect of berberine on the differentiation of adipocyte," Zhonghua Yi Xue Za Zhi, vol. 83, no. 4, pp. 338-340, 2003 (Chinese).

[16] L. B. Zhou, M. D. Chen, H. D. Song et al., "Effect of berberine on leptin and resistin gene expression of fat cells," Chinese Journal of Internal Medicine, vol. 43, no. 1, pp. 56-57, 2004 (Chinese).

[17] W. Gu, W.-H. Zeng, and H.-Y. Hu, "Effects of berberine on adiponectin mRNA expression in 3T3-L1 adipocyte," China Journal of Chinese Materia Medical, vol. 30, no. 4, pp. 286-288, 2005.

[18] Q. Zhang, X. Xiao, K. Feng et al., "Berberine moderates glucose and lipid metabolism through multipathway mechanism," Evidence-Based Complementary and Alternative Medicine, vol. 2011, Article ID 924851, 10 pages, 2011.

[19] J. H. Yin, M. Li, J. Yang, and C. Y. Wu, "Primary culture of human omental preadipocytes and study of their biological properties," Zhonghua Yi Xue Za Zhi, vol. 87, no. 12, pp. 838841, 2007 (Chinese).

[20] L. H. Liu, X. K. Wang, Y. D. Hu, J. L. Kang, L. L. Wang, and S. Li, "Effects of a fatty acid synthase inhibitor on adipocyte differentiation of mouse 3T3-L1 cells," Acta Pharmacologica Sinica, vol. 25, no. 8, pp. 1052-1057, 2004. 
[21] Z. W. Wang, X. Wang, X. Li et al., "Prevalence and trend of metabolic syndrome in middle-aged Chinese population," Zhonghua Liu Xing Bing Xue Za Zhi, vol. 30, no. 6, pp. 596600, 2009 (Chinese).

[22] L. B. Zhou, M. D. Chen, H. D. Song et al., "Effect of berberine on glucose transport in adipocyte and its mechanism," Chinese Journal of Endorcrinology and Metabolism, vol. 19, no. 6, pp. 38-41, 2003.

[23] J. Yin, H. Xing, and J. Ye, "Efficacy of berberine in patients with type 2 diabetes mellitus," Metabolism, vol. 57, no. 5, pp. 712-717, 2008.

[24] T. McLaughlin, A. Sherman, P. Tsao et al., "Enhanced proportion of small adipose cells in insulin-resistant vs insulinsensitive obese individuals implicates impaired adipogenesis," Diabetologia, vol. 50, no. 8, pp. 1707-1715, 2007.

[25] E. D. Rosen and O. A. MacDougald, "Adipocyte differentiation from the inside out," Nature Reviews Molecular Cell Biology, vol. 7, no. 12, pp. 885-896, 2006.

[26] E. D. Rosen, C. J. Walkey, P. Puigserver, and B. M. Spiegelman, "Transcriptional regulation of adipogenesis," Genes and Development, vol. 14, no. 11, pp. 1293-1307, 2000.

[27] S. R. Farmer, "Transcriptional control of adipocyte formation," Cell Metabolism, vol. 4, no. 4, pp. 263-273, 2006.

[28] Y. S. Lee, W. S. Kim, K. H. Kim et al., "Berberine, a natural plant product, activates AMP-activated protein kinase with beneficial metabolic effects in diabetic and insulin-resistant states," Diabetes, vol. 55, no. 8, pp. 2256-2264, 2006.

[29] C. Huang, Y. Zhang, Z. Gong et al., "Berberine inhibits 3T3L1 adipocyte differentiation through the PPAR $\gamma$ pathway," Biochemical and Biophysical Research Communications, vol. 348, no. 2, pp. 571-578, 2006.

[30] Q. Tong, G. Dalgin, H. Xu, C. N. Ting, J. M. Leiden, and G. S. Hotamisligil, "Function of GATA transcription factors in preadipocyte-adipocyte transition," Science, vol. 290, no. 5489, pp. $134-138,2000$.

[31] Q. Tong, J. Tsai, G. Tan, G. Dalgin, and G. S. Hotamisligil, "Interaction between GATA and the C/EBP family of transcription factors is critical in GATA-mediated suppression of adipocyte differentiation," Molecular and Cellular Biology, vol. 25, no. 2, pp. 706-715, 2005.

[32] Y. Hu and G. E. Davies, "Berberine increases expression of GATA-2 and GATA-3 during inhibition of adipocyte differentiation," Phytomedicine, vol. 16, no. 9, pp. 864-873, 2009.

[33] Y. Hu, H. Fahmy, J. K. Zjawiony, and G. E. Davies, “Inhibitory effect and transcriptional impact of berberine and evodiamine on human white preadipocyte differentiation," Fitoterapia, vol. 81, no. 4, pp. 259-268, 2010.

[34] W. Kong, J. Wei, P. Abidi et al., "Berberine is a novel cholesterol-lowering drug working through a unique mechanism distinct from statins," Nature Medicine, vol. 10, no. 12, pp. 1344-1351, 2004.

[35] N. Oda, S. Imamura, T. Fujita et al., "The ratio of leptin to adiponectin can be used as an index of insulin resistance," Metabolism, vol. 57, no. 2, pp. 268-273, 2008. 


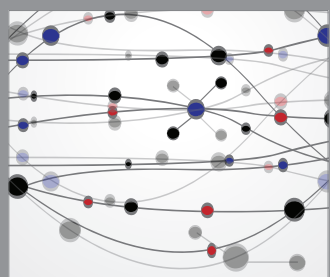

The Scientific World Journal
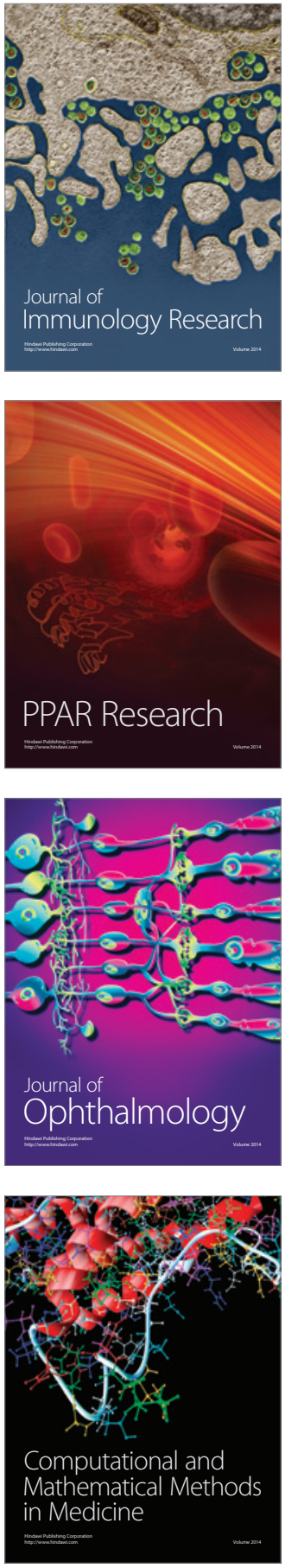

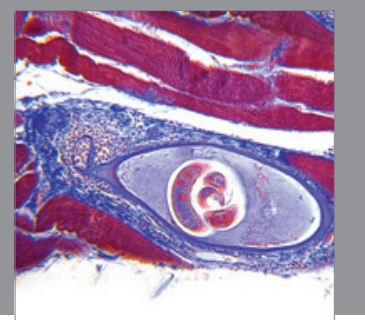

Gastroenterology

Research and Practice
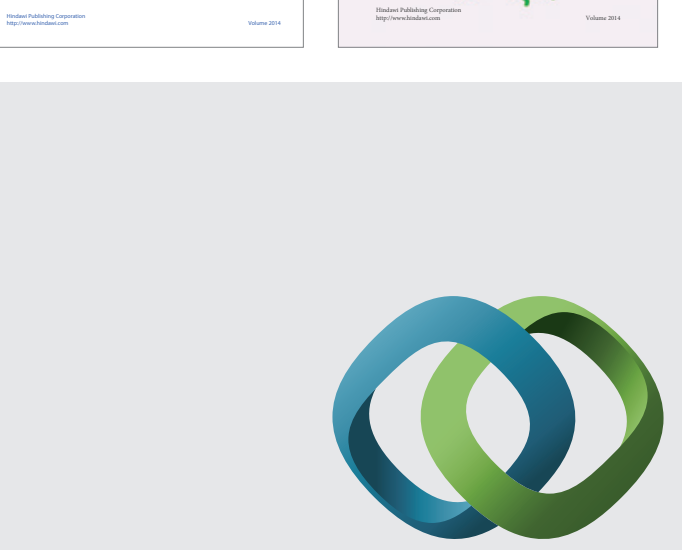

\section{Hindawi}

Submit your manuscripts at

http://www.hindawi.com
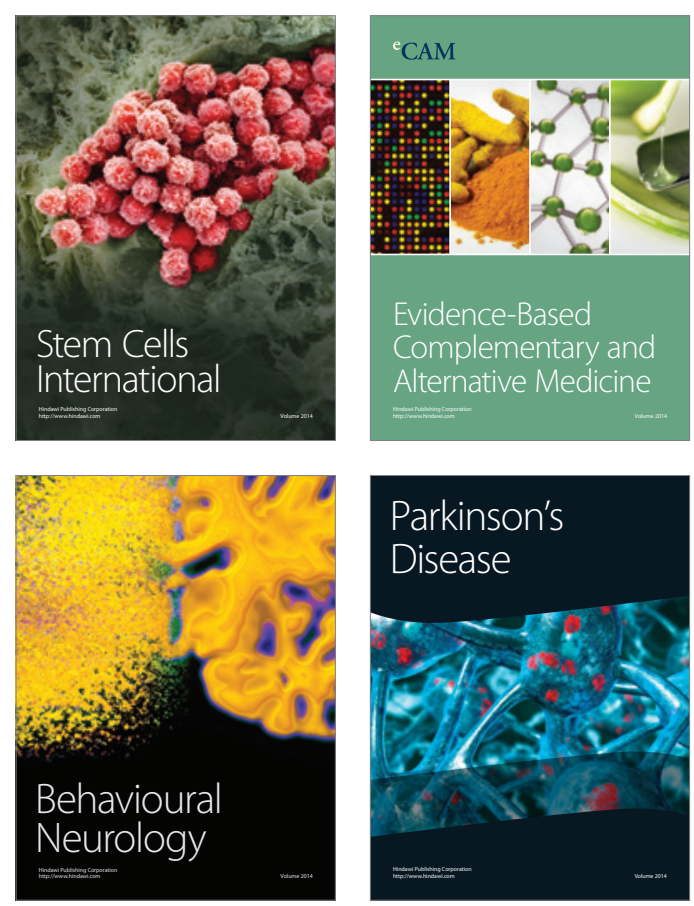

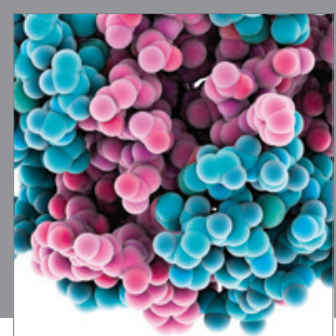

Journal of
Diabetes Research

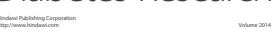

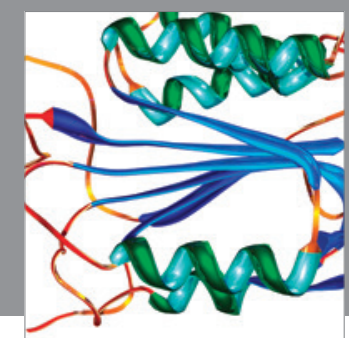

Disease Markers
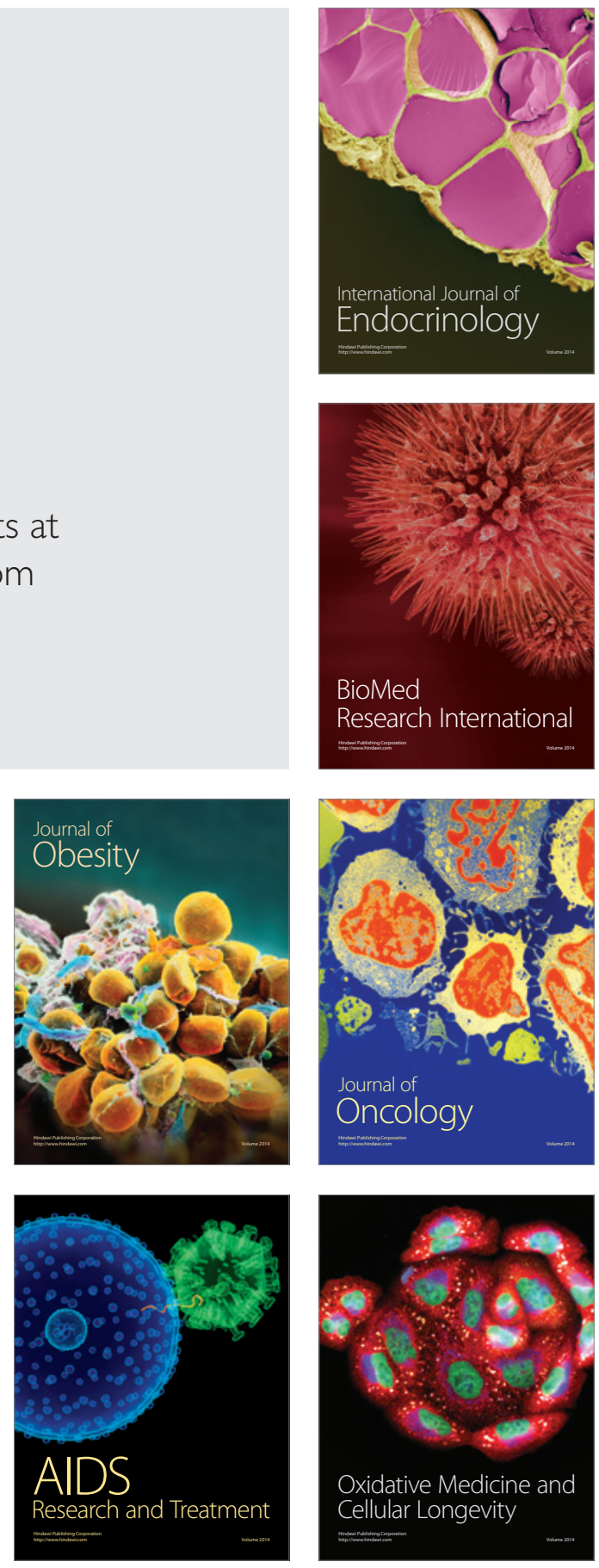